INVESTIGACIÓN/RESEARCH

\title{
EL RETO DE CARLOS SORIA (I): LA CREACIÓN DE UN FENÓMENO EN REDES SOCIALES
}

Juan Enrique Gonzálvez Vallés1: Universidad Camilo José Cela. España. juanen2012@gmail.com

\section{RESUMEN}

La presencia de Carlos Soria en las redes sociales ha causado toda una revolución en Facebook, Twitter y YouTube. Tras la decisión de BBVA de patrocinar al alpinista de 73 años se puso en marcha una maquinaria bien engrasada que ayudaría a trasmitir la imagen conjunta BBVA-Carlos Soria. De esta forma, el alpinista español obtenía el respaldo de una gran empresa que le ayude a completar su reto de coronar las catorce montañas más altas de la Tierra; y el banco tenía la oportunidad de asociar su imagen a un proyecto que le ayudase a mejorar la impresión que los clientes, actuales y potenciales, tienen sobre esta entidad bancaria en particular, y sobre los bancos en general.

PALABRAS CLAVE: Carlos Soria - BBVA - Redes sociales - Patrocinio - Perfiles.

\footnotetext{
${ }^{1}$ Autor correspondiente:

Juan Enrique Gonzálvez Vallés: Profesor del centro de estudios Formatik, asociado a la Universidad Camilo José Cela. Miembro del Grupo de Investigación Concilium de la Universidad Complutense de Madrid. Editor adjunto de las Revistas científicas SEECI y Vivat Academia. España.

Correo: juanen2012@gmail.com
} 


\title{
THE CHALLENGE OF CARLOS SORIA (I): THE CREATION OF A PHENOMENON IN NETWORKS
}

\begin{abstract}
Carlos Soria's presence on social networking has caused a revolution in Facebook, Twitter and YouTube. Following the decision to sponsor the climber BBVA of 73 years, a well-oiled machine was launched that would help to pass on the joint image BBVACarlos Soria. Thus, the Spanish climber got the backing of a large company to help him to complete his challenge to crown the fourteen highest mountains on Earth, and the bank had the opportunity to associate its image with a project that help it to improve the impression that customers have about this particular bank, and banks in general.
\end{abstract}

KEY WORDS: Carlos Soria - BBVA - Social Networking - Sponsorship - Profiles.

\section{INTRODUCCIÓN}

No hace falta acudir a un entorno exterior lejano para constatar que las redes sociales están cambiando la vida de todos. Se convocan manifestaciones a favor y en contra de determinadas corrientes ideológicas, el fenómeno fan es capaz de colapsar la red a través de mensajes digitales que se transfoman en colapsos en las calles y plazas para idolatrar a los nuevos becerros de oro, construidos a base de marketing y capital, si es que es posible disasociar ambos conceptos.

Desde la plaza Tahrir hasta la Puerta del Sol, pasando por el fenómeno de Barack Obama o la declaración del estado de emergencia en Noruega por la presencia del cantante Justin Bieber, la presencia y el poder de las redes sociales se ha hecho cada vez más patente en el mundo que todos habitamos. Movilizan más que cualquier revolución analógica que hayamos podido presenciar y el ruido que generan, plasmado de manera mediática fundamentalmente, mueven a la sociedad en todos los ámbitos, desde los más trascendentales hasta los más superficiales.

Con este caldo de cultivo, las redes sociales han encontrado en el deporte un ámbito perfecto para su propagación. Efecto que también se producido en el sentido contrario, puesto que el deporte se ha percatado de las grandes capacidades de las redes sociales para transmitir su mensaje, acercarse al ciudadano medio y ha encontrado un medio neutro, alejado de las tendencias de los grandes medios pertenecientes a los grandes grupos empresariales.

No ha sido extraño, pues, que los deportes mayoritarios y las estrellas de los mismos hayan puesto sus ojos sobre las redes sociales. Como apuntábamos antes, aquí han encontrado un canal para transmitir su mensaje sin filtros de ningún tipo ni interpretaciones previas. Desde el pedestal donde están subidos, estos personajes o 
instituciones pueden mostrarse cercanos a sus seguidores alrededor de todo el mensaje, construyendo un ecosistema favorable para sus intereses.

Lo más sorprendente ha sido como los deportes minoritarios han encontrado también su hueco y, sobre todo, una mayor repercusión de su actividad. Raro es el club de fútbol de división humilde, club de patinaje, petanca o dominó que no posee su propio grupo de Facebook, cuenta de Twitter o perfil de Google+. El marketing relacional ha ayudado a que, al menos, se constituyan pequeños grupos o grupúsculos de familiares y amigos que tengan noticias actualizadas sobre estos equipos que antes desarrollaban su actividad en la más estricta intimidad.

Sin embargo, el fenómeno que vamos a estudiar no encaja en ninguno de los dos grupos anteriores. O más bien encaja en ambos y ayuda a crear un grupo nuevo. Cuando BBVA comenzó a patrocinar al montañero Carlos Soria gestó un proyecto para asociar su imagen a la de uno de los alpinistas más respetados, pero los resultados que consiguió estuvieron muy por encima de lo que podían esperar.

\section{METODOLOGÍA}

Nuestra intención es la de que se constate y demuestre que la presencia de una sinergia entre BBVA y Carlos Soria, a través de una herramienta de las relaciones públicas externas como es el patrocinio, ha provocado que se desate en las redes sociales todo un fenómeno singular e inigualable, sobre todo partiendo de la base de que el alpinismo es un deporte minoritario.

Además, también tendremos presente la repercusión que se ha producido en los medios de comunicación, tanto analógicos como digitales. Y, por supuesto, las conexiones que se han producido entre todos estos canales, puesto que los reclamos entre unos y otros han sido lo que han producido gran parte del éxito de este caso, sobre todo por la viralidad provocada.

Para ello, realizaremos un análisis de los datos obtenidos tras el seguimiento pormenorizado de los perfiles de Carlos Soria en Facebook, Twitter y Youtube. Consideramos que el método expositivo y demostrativo es el más adecuado para el caso que nos ocupa, puesto que nos ayudará a obtener las conclusiones pertinentes. Además, expondremos los casos más reseñables dentro de los citados perfiles, como muestra inequívoca de lo que el público ha acogido como contenidos preferidos y predilectos.

Resulta relevante conocer todos estos puntos puesto que podremos entonces evaluar la sostenibilidad del proyecto $\mathrm{y}$, en caso positivo, desgranar las posibles acciones futuras que se puedan llevar a cabo. Buscando, en definitiva, obtener los resultados positivos que, además, puedan ser aplicables a otros proyectos tanto dentro del mundo del deporte como en lo relacionado con otros patrocinios que ésta u otras entidades deseen llevar a cabo. 
Estableciendo con claridad nuestro objetivo, estudiaremos la comunicación y la gestión de la misma que sea realizan en el proyecto de patrocinio de BBVA con Carlos Soria, focalizando sobre el estudio en las redes sociales. Al contar con tres perfiles activos (Facebook, Twitter y Youtube) podremos contemplar las diferencias fundamentales entre ellos en aspectos o indicadores de importancia como el número de seguidores, el número de impactos o el número de publicaciones en cada uno de ellos.

Con el establecimiento de los análisis que acabamos de citar también seremos testigos de cómo funcionan individualmente cada uno de estos perfiles y su relación o relaciones con sus adeptos, llámense fans, followers o suscriptores. Partiremos de la base de que en todos los perfiles se intenta transmitir siempre de la mejor manera posible, con el objetivo último de crear una imagen positiva, de la cual se beneficien los dos actores de este patrocinio, esto es, BBVA y Carlos Soria.

\section{ANÁLISIS Y DISCUSIÓN}

\subsection{BBVA}

La situación económica actual ha hecho que la imagen de las entidades bancarias se deteriore, al ser consideradas como las culpables de la misma. Resultando cierta o no esta afirmación, el lastre en términos de imagen para los bancos es patente. De hecho, podemos encontrar hasta encuestas que parten de organismos oficiales que dan fe de que los españoles ponen en su punto de mira a los bancos para buscar responsables de la actual situación económica.

El Centro de Investigaciones Sociológicas (CIS) elaboró en abril de 2012 una consulta para buscar a los responsables de la crisis entre la ciudadanía española. El gobierno de España resultó ser el triste ganador, con más del $30 \%$ de los resultados a su favor. Con menos de seis puntos de desventaja $(23,6 \%)$, los bancos se colgaron la medalla de plata de un podio que se cerraba con el $21,7 \%$ de la situación económico internacional. 
Gráfico 1. Encuesta sobre la responsabilidad de la crisis.

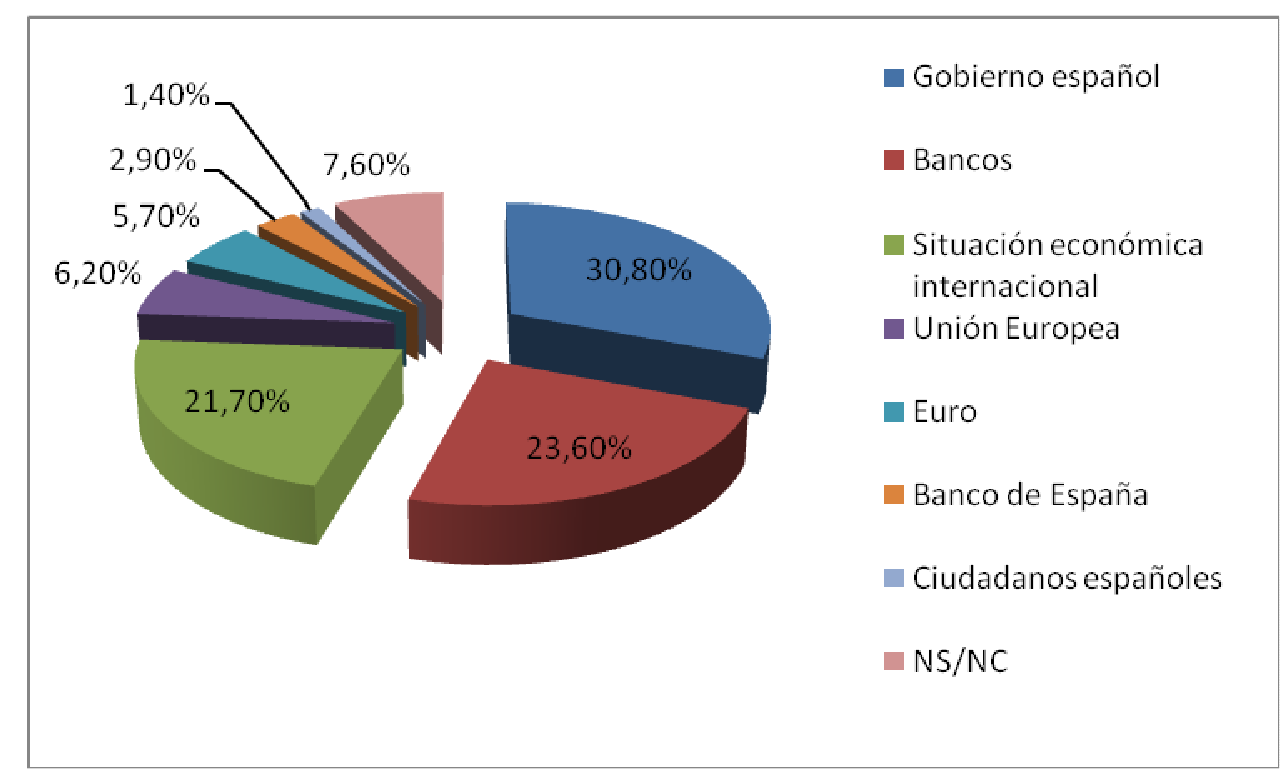

Fuente: Centro de Investigaciones Sociológicas

\subsubsection{Liga BBVA y Liga Adelante.}

Ante esta realidad, real o artificial, no entramos en esa discusión, las entidades bancarias buscan los mecanismos o herramientas que les sean más útiles para contrarrestar esta pérdida de imagen. Dentro de las relaciones públicas externas, el patrocinio ha sido tradicionalmente uno de esos mecanismos que mejor resultado ha proporcionado. Es por ello que, al igual que otras entidades bancarias, BBVA ha ejercido esta opción sobre un elemento con connotaciones tan positivas como es el mundo del deporte.

Sus principales apuestas han venido de los deportes mayoritarios y de las estrellas que en ellos militan. Así, en acuerdo con la Liga de Fútbol Profesional, BBVA pasó a ser el principal patrocinador de la Liga española, que pasó a llamarse Liga BBVA en su Primera División; y Liga Adelante, en su Segunda División. En este caso se referencia la marca a la máxima categoría del fútbol español, mientras que el eslogan principal de la entidad queda como reclamo o claim del segundo escalón.

\section{Foto 1. Logos Liga BBVA y Liga Adelante.}

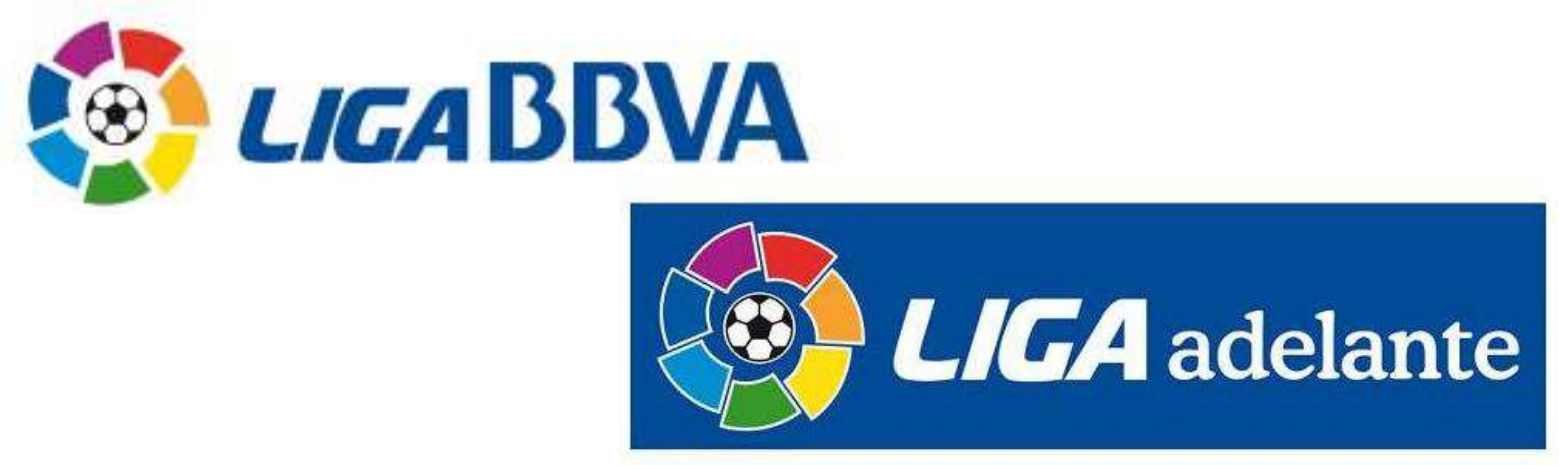

Fuente: BBVA 
En este mismo sentido, BBVA buscó unos embajadores de marca mundiales que también poseyeran una reconocida e irrefutable buena imagen con la que poder asociarse. La lista quedó acotada por encuestas y consultas que permitían escoger a los preferidos por los españoles. Los diez primeros en la lista fueron los siguientes:

1. Rafa Nadal.

2. Pau Gasol.

3. Andrés Iniesta.

4. Paco León.

5. Miguel Indurain.

6. Xavi Hernández.

7. Pep Guardiola.

8. Vicente del Bosque.

9. Iker Casillas.

10. Matías Prats.

Había referenciado ya su imagen a la del fútbol español, BBVA decidió nombrar como embajadores de marca a Andrés Iniesta y a Iker Casillas. Dos jóvenes deportistas, muy valorados por la gente y pertenecientes a los dos equipos grandes del fútbol español, Barcelona y Real Madrid, Real Madrid y Barcelona.

\section{Foto 2. Iker Casillas y Andrés Iniesta, embajadores de marca BBVA.}

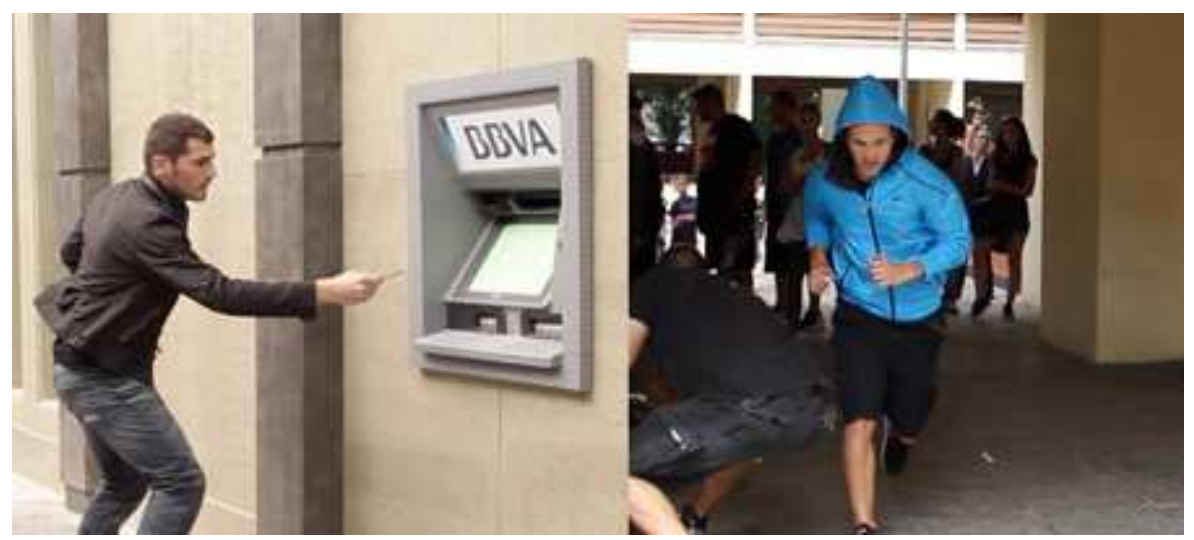

Fuente: BBVA

\subsubsection{NBA Official Bank.}

La segunda apuesta poseía un valor estratégico muy importante. BBVA llegó a un acuerdo con la NBA para el ser el banco oficial de la prestigiosa liga de baloncesto estadounidense. No se ejercía un patrocinio con una presencia tan importante como en el caso de Liga BBVA, pero se implantaba la marca BBVA para un entorno de influencia claro y amplio, puesto que abarcaba desde los propios Estados Unidos, donde BBVA está presente, hasta su repercusión en los países sudamericanos, donde la NBA es seguida e idolatrada, incluso más que la liga de fútbol española. 
Foto 3. Logo patrocinio BBVA.

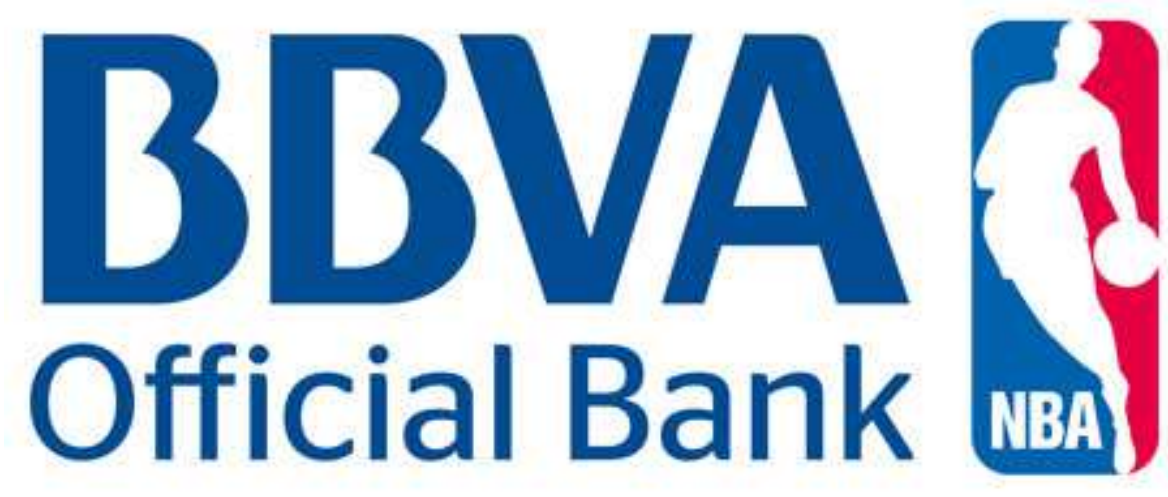

Fuente: BBVA

\subsubsection{Otros patrocinios.}

Al margen de los patrocinios que acabamos de ver, relacionados con el mundo del deporte, y del que estudiaremos particularmente, como es el caso de Carlos Soria, BBVA no ha renunciado a otro tipo de patrocinios, que también forman parte de la buena imagen que el banco pretende dar de sí mismo. Entre ellos:

1. Ruta Quetzal BBVA.

2. Museo Nacional del Prado.

3. Fundación Joan Miró de Barcelona.

4. Museo Guggenheim de Bilbao.

5. Fundación Albéniz y Escuela Superior de Música Reina Sofía.

6. Fundéu BBVA.

7. Blue BBVA.

8. ...

\subsection{Carlos Soria.}

Carlos Soria Fontán nació en Ávila el 5 de febrero de 1939 y es uno de los alpinistas españoles con mayor prestigio. Con 73 años está llevando a cabo el reto de ser la persona de más edad en lograr la cima en las catorce montañas más altas del mundo. Soria es la única persona en el mundo que ha escalado nueve ochomiles con más de sesenta años, y tres de ellos con más de setenta. Se trata también de la persona más longeva en alcanzar la cumbre del K2 (65 años), Broad Peak (68 años), Makalu (69 años), Gasherbrum I (70 años) y Manaslu (71 años). 


\section{Gráfico 2. Ascensiones por orden cronológico.}

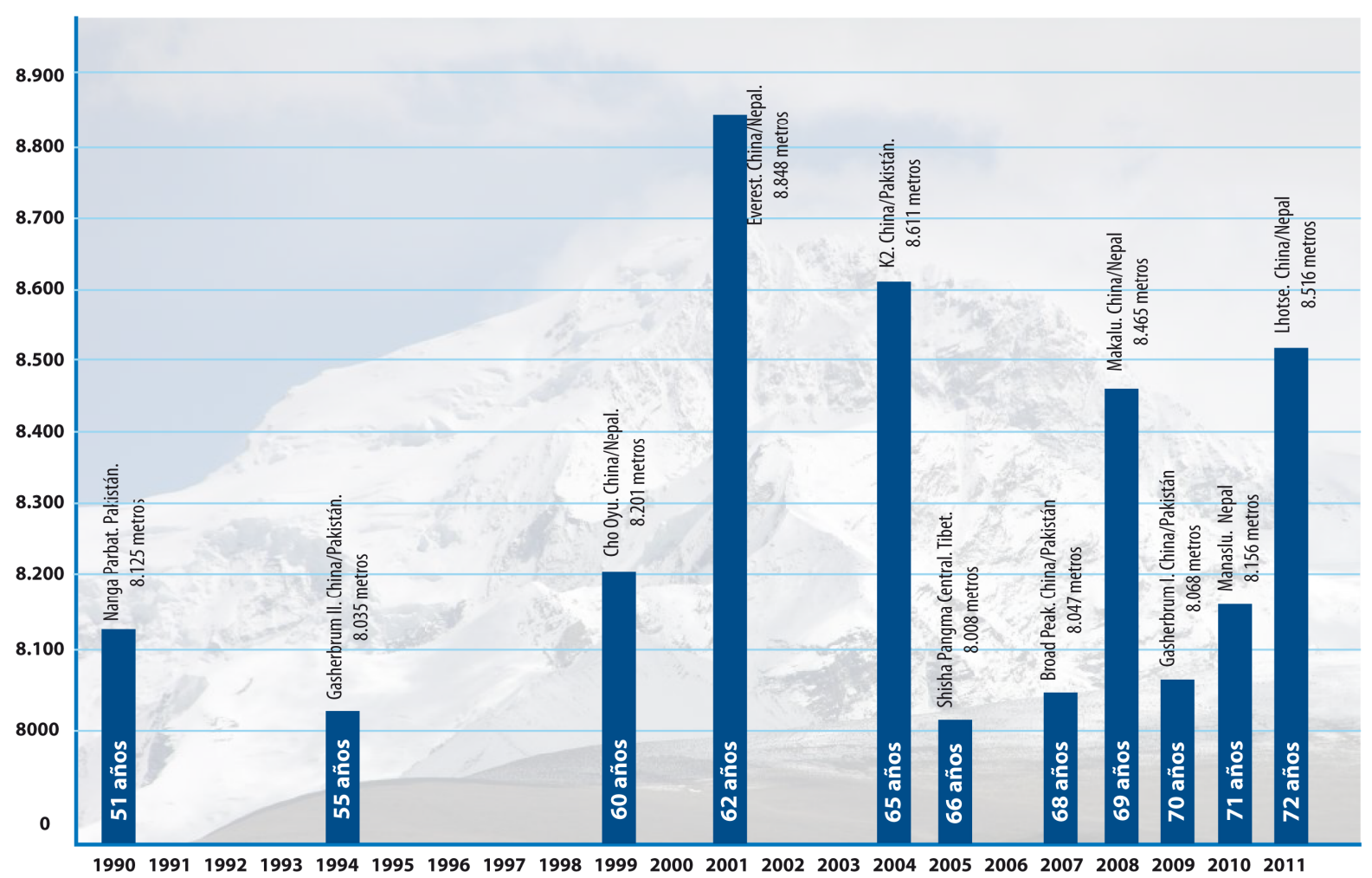

Fuente: BBVA

Carlos Soria se ha convertido en un referente para todos aquellos que consideran que la edad no debe convertirse en un obstáculo para cumplir todos los objetivos que te propongas. Su tenacidad y espíritu de superación han inspirado a muchas personas para disfrutar de una edad avanzada pero sin complejo y con ganas de disfrutar al máximo de todo lo que pueda.

El historial de Carlos Soria se remonta a cuando, con catorce años, realizó su primera excursión a la Sierra de Guadarrama en Madrid. Desde ahí, sus viajes han sido casi innumerables, desde 1968, cuando formó parte de la primera expedición española que viajó a Rusia para subir el monte Elbrus, hasta 1973, cuando también formó parte de la primera expedición española que viajó al Himalaya para tratar de coronar el Manaslu, hecho que él no conseguiría hasta 37 años después, en 2010.

La mayoría de las ocasiones en que Carlos Soria ha estado de expedición en el Himalaya lo ha hecho solo, acompañado únicamente de algún sherpa y/o algún porteador. Desde que cuenta con el patrocinio de BBVA, Soria lidera su propia expedición, con la presencia de un médico, cámaras y varios sherpas y porteadores que le ayudan en la consecución de su objetivo.

Carlos Soria se ha convertido en una de las personalidades más respetadas y queridas del mundo del deporte en general, y del alpinismo en particular. Su espíritu humilde y 
sus grandes retos y hazañas son más propias de espectadores del deporte que de un actor protagonista.

\subsection{BBVA-Carlos Soria.}

A finales de agosto de 2011, BBVA y Carlos Soria establecen una alianza estratégica por la cual la entidad bancaria colaborará con el alpinista español mediante la fórmula del patrocinio para su objetivo de lograr coronar las catorce montañas más altas de la Tierra. Una apuesta por, como ya hemos visto en el apartado anterior, un deportista con valores. Algo que destacó el consejero delegado de BBVA, Ángel Cano, en la presentación de la expedición al Annapurna en 2012.

\section{Foto 4. Ángel Cano (consejero delegado BBVA) y Carlos Soria.}

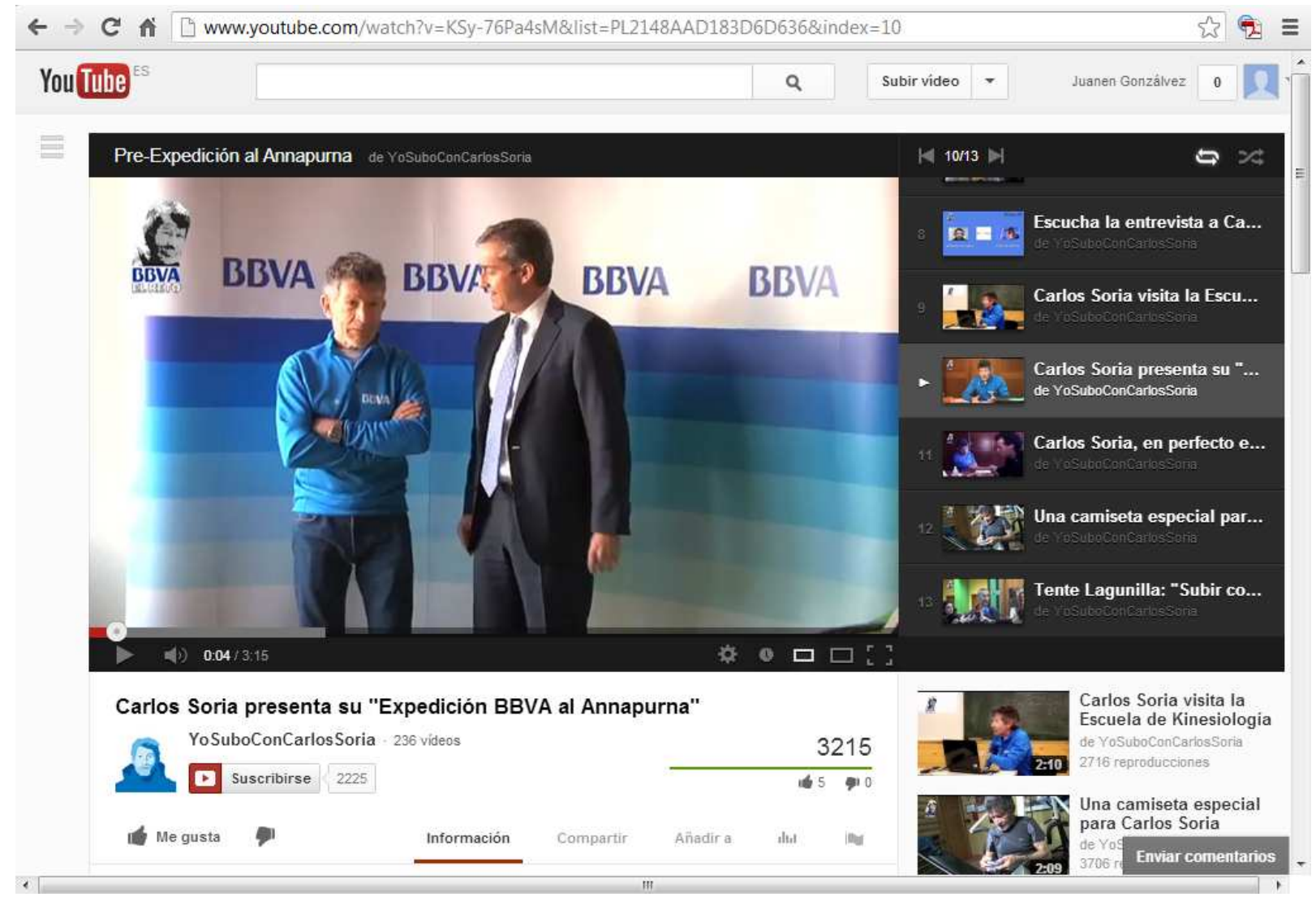

Fuente: YouTube: http://www.youtube.com/watch?v=KSy-76Pa4sM

Esta asociación sin duda beneficiaba a los dos actores principales del patrocinio pero había que elevar al gran público esta idea para que tuviera el grado de conocimiento y, por tanto, de afectación adecuado. Por ello, BBVA puso su empeño en las redes sociales para llevar a cabo esta labor, focalizando su esfuerzo en Facebook, Twitter y YouTube.

\subsubsection{Facebook.}

Sin duda, la más exitosa de las redes sociales, por lo menos en el caso de BBVA-Carlos Soria. A pesar de que el alpinista poseía una cuenta personal 
(http://www.facebook.com/carlos.soriafontan) se creó una página como personaje público (http://www.facebook.com/yosuboconcarlossoria) con mucho éxito desde su génesis a finales de agosto de 2011.

\section{Foto 4. Perfil de Facebook "Yo Subo con Carlos Soria".}

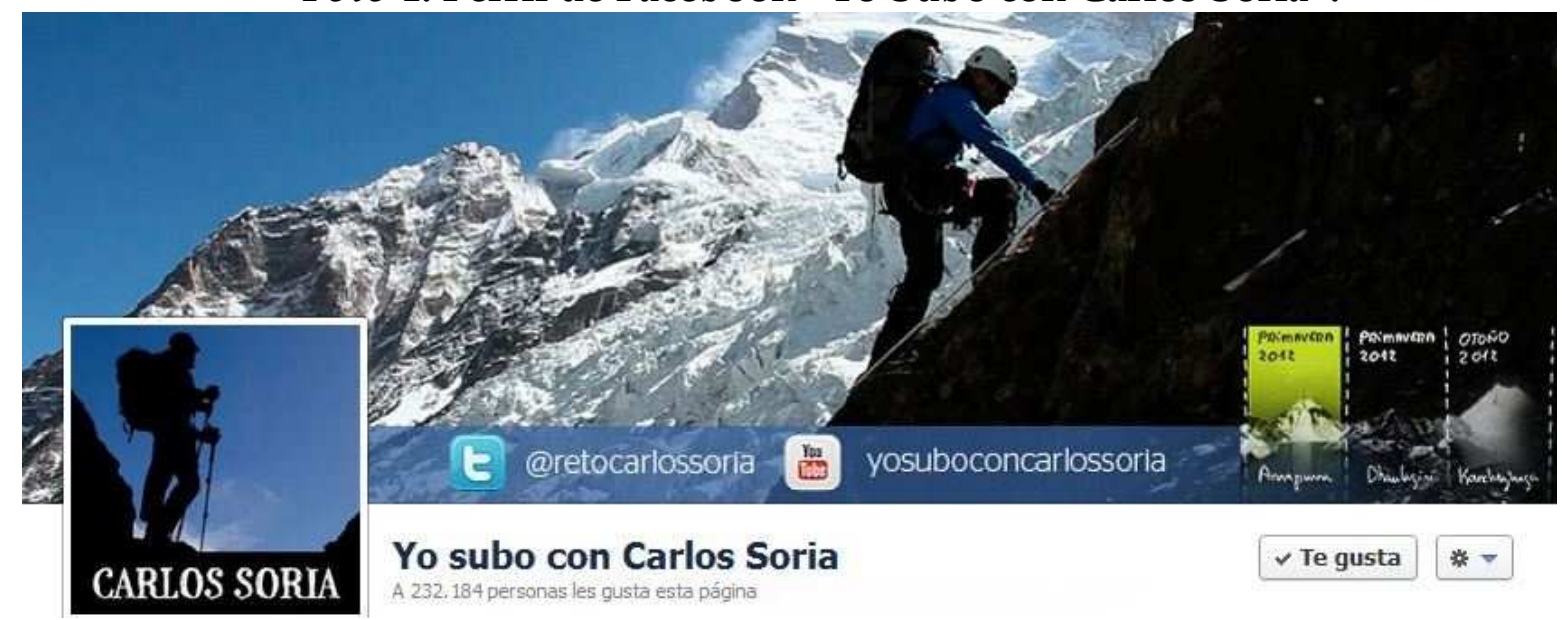

Fuente: Facebook.

A continuación, y como haremos también con los perfile de Twitter y YouTube, expondremos los datos recogidos durante un año de seguimiento al que ya se puede calificar como 'fenómeno Carlos Soria'. En primer lugar, el histórico de los seguidores en Facebook del perfil creado por BBVA, que ha marcado un hito en cuanto al seguimiento de personajes públicos se refiere:

\section{Gráfico 3. Histórico seguidores "Yo Subo con Carlos Soria".}

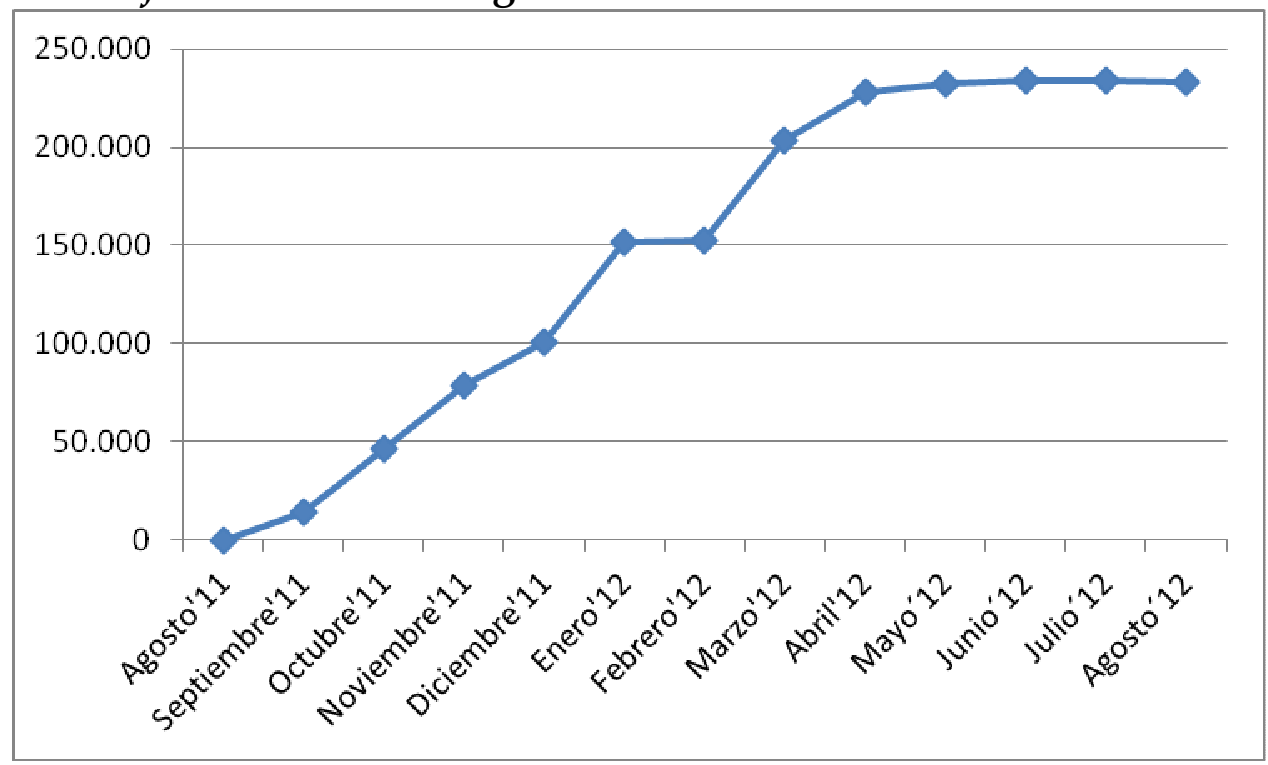

Fuente: Facebook.

El análisis de este gráfico nos lleva a utilizar la palabra impresionante en primer lugar. En menos de seis meses, "Yo Subo con Carlos Soria" había logrado más de 100.000 seguidores, algo al alcance de pocos perfiles de personajes conocidos. Tenemos en 
cuenta que, entre medias, Carlos Soria y sus compañeros acometieron una primera expedición, ya bajo el paraguas de BBVA, tratando de coronar el Dhaulagiri

Este hecho, que se produjo fundamentalmente entre los meses de septiembre y octubre de 2011, produjo sin duda un efecto llamada ante la cantidad y calidad de contenidos aportados en dicho perfil. No entraremos en un análisis pormenorizado de los mismos puesto que eso será objeto de otro investigación posterior, pero sí que apuntamos que la gran cantidad y calidad de las fotografía, vídeos y comunicaciones aportadas supusieron un magnífico reclamo para crear un efecto viral que aportó seguidores a este perfil.

El segundo punto fuerte del análisis de este histórico llega en un periodo que podemos considerar como 'valle', puesto que entre noviembre y marzo no existe ninguna expedición por parte de Carlos Soria al Himalaya. Sin embargo, la continua actualización del perfil con nuevos contenidos, unido a la presencia de Carlos Soria en medios de comunicación, estrategia complementaria pero sin duda muy efectiva, logró que los seguidores continuaran incrementándose de forma espectacular.

El tercer aumento importante de seguidores se produjo durante la expedición de Carlos Soria al Annapurna. Considerado como normal, los seguidores aumentaron al volver a producirse el momento clímax que todos los seguidores estaban esperando, fidelizando a los actuales y aumentando la viralidad que produjo un mayor número de nuevas incorporaciones. Es lógico que, al ofrecer de nuevo contenidos de alta calidad y, sobre todo, totalmente diferenciados del casi el $100 \%$ de otros personajes públicos, exceptuando otros alpinistas, se produjera este incremento que dejó los seguidores superando los 233.000 en el mes de junio de 2012.

A partir de ese momento se ha producido un estancamiento en el número de seguidores de este perfil en Facebook. Queda saber, quizá en un análisis posterior, si los dígitos han llegado ya a su tope y es difícil aumentarlos o una nueva expedición sería capaz de aumentarlos todavía más.

\subsubsection{Twitter.}

El principal damnificado del esfuerzo hecho en Facebook, o mejor dicho para Facebook, fue el perfil de Twitter, con un éxito sólo relativo. 
Gráfico 4. Histórico followers "@RetoCarlosSoria".

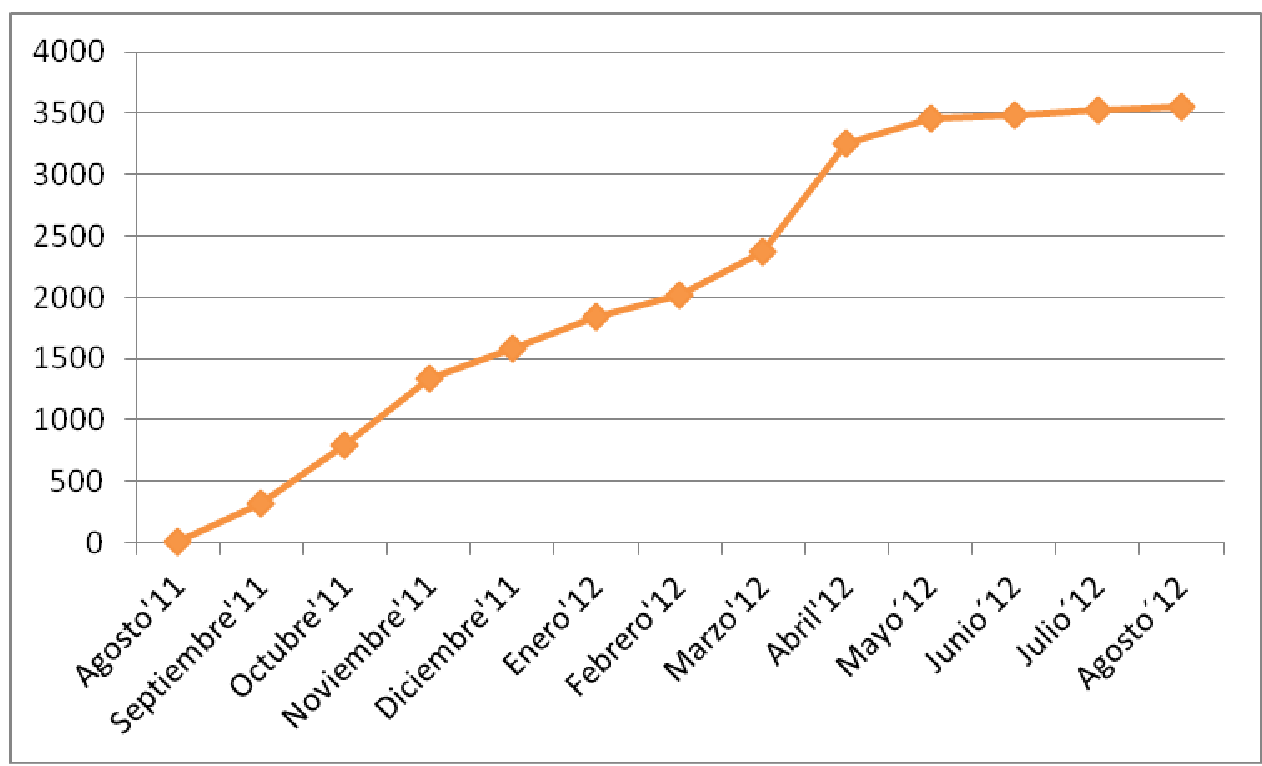

Fuente: Twitter.

Al igual que ocurría con Facebook, Carlos Soria ya poseía una cuenta personal en Twitter (@carlossfontan) pero BBVA se decidió a crear un perfil oficial (@RetoCarlosSoria) para contar todas las novedades sobre el alpinista y sus expediciones. Los contenidos seguían los que se publicaban en Facebook y la mayoría de ellos se referenciaban a esta red social.

El principal problema con el que se ha encontrado este perfil es el de no poder anotar un seguimiento más continuo que fueran más en la línea de lo que esta red social consume. Al poseer una temporalidad más alta, puesto que en los timelines de los perfiles de los usuarios de Twitter los mensajes aparecen y son sustituidos por otros nuevos con más frecuencia, la no repetición de los mensajes o la creación de mensajes novedosos hacen perder atractivo a esta cuenta.

Aun así, el aumento ha sido sostenido durante estos primeros doce meses, destacando un fuerte aumento durante la expedición primaveral al Annapurna, con más de 600 nuevos followers. Los números son discretos, pero el perfil en Twitter ya cuenta con más de 3.500 followers y en ningún mes se ha producido un descenso de seguidores, con lo que se espera que dentro del segundo año puedan alcanzar números significativos.

\subsubsection{YouTube.}

El principal contenedor de vídeos del mundo ha sido utilizado más como apoyo de los otros dos perfiles y, sobre todo, de Facebook, que como otra red social en busca de nuevo seguidores. Los suscriptores no han sido demasiado numerosos, tal y como lo reflejan las estadísticas recopiladas. 


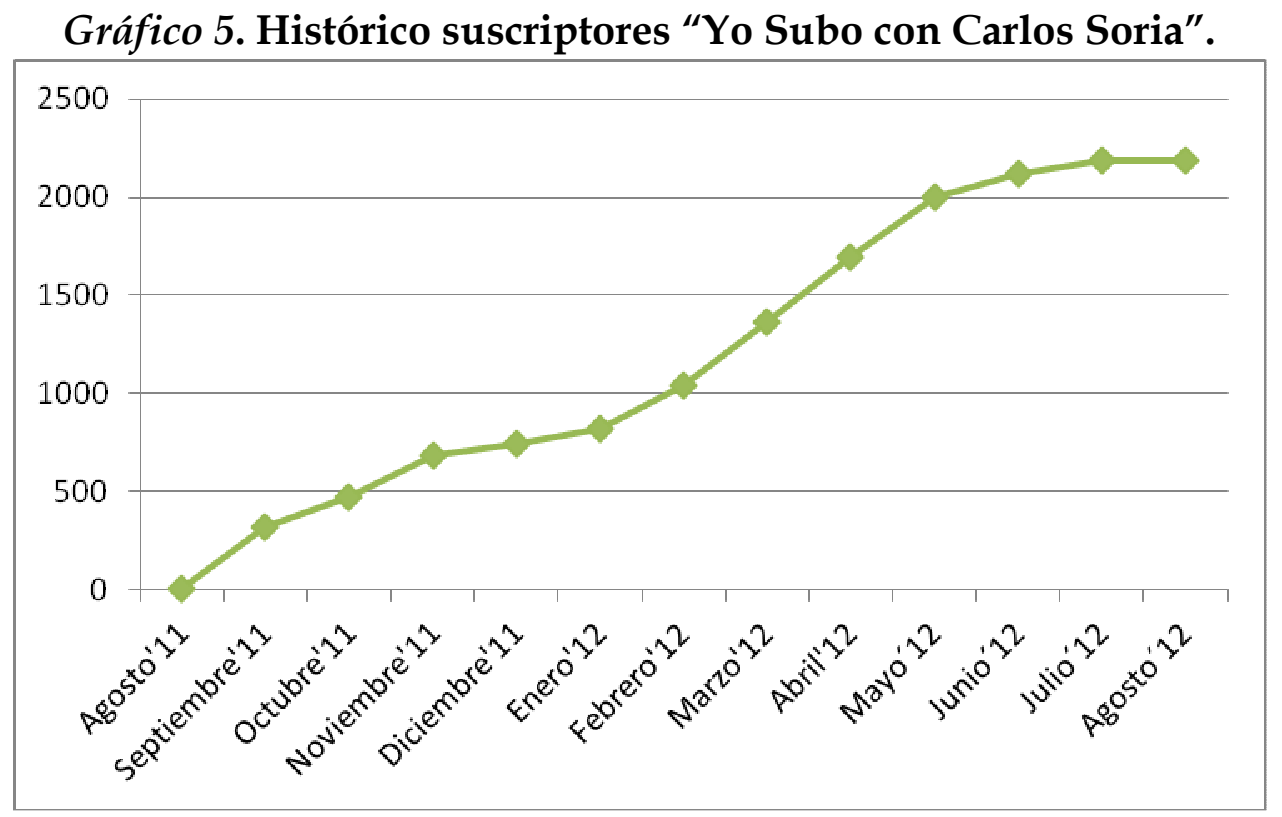

Fuente: YouTube.

El número de suscriptores es poco significativo pero, sin embargo, queda para un estudio posterior la importancia que los vídeos proporcionados por este canal han tenido en el éxito global de este patrocinio. Simplemente apuntaremos que hasta agosto de 2012 se habían superado las 300 piezas audiovisuales, superando las 900.000 reproducciones. Un pequeño apunte de lo beneficioso que ha sido YouTube para la estrategia de comunicación de patrocinio ejercida por BBVA.

\section{CONCLUSIONES}

Los resultados de esta investigación ponen de manifiesto que la estrategia de patrocinio de BBVA-Carlos Soria en redes sociales ha sido un acierto y exitosa de todo punto. Hemos constatado como la entidad bancaria poseía un problema de imagen, fruto de la crisis económica actual. Como los españoles creen que los bancos son parte del problema, más que de la solución para el estado actual del país.

Fruto de ello fue la búsqueda de un patrocinio que llevara asociados valores positivos y que encajara con la política de patrocinios de BBVA, focalizada en el deporte. En este sentido, el acuerdo con la Liga de Fútbol Profesional para patrocinar lo que pasaría a llamarse la Liga BBVA; y el acuerdo con la NBA para ser el banco, resultaron excelentes bancos de pruebas para buscar otro acuerdo en el mismo campo.

Carlos Soria ha resultado ser, no sólo una apuesta ganadora, sino que ha pasado a convertirse en todo un símbolo de para BBVA, que lo elevó a la categoría de embajador mundial de su marca. No es que por este patrocinio se haya revertido la imagen que BBVA tenía pero el interés demostrado por el alto número de seguidores sí que supone un espacio de promoción perfecto para transmitir lo valores que la entidad bancaria desea sobre sí misma. 
Con casi 250.000 seguidores, aunando los de los tres perfiles de Facebook, Twitter y YouTube, Carlos Soria se ha convertido en un fenómeno mediático muy por encima de cualquier expectativa inicial, y superando a muchos de los más afamados deportistas españoles, y parte también de los extranjeros. En este punto, queda por analizar el origen geográfico de los seguidores, que será objeto de análisis de un artículo de investigación posterior.

El mayor esfuerzo en las redes sociales se ha producido, sin lugar a dudas, en Facebook. La inclusión de contenidos que se adaptan a las características de esta red social han supuesto un boom que llevó al perfil "Yo Subo con Carlos Soria" a superar los 230.000 seguidores en menos de un año. El esfuerzo, sin duda, transcurrido este primer año será el de fidelizar esta cantidad ingente de personas.

El resto de perfiles han tenido un éxito menor, puesto que no cabe calificar el número de seguidores de Twitter y de YouTube como un fracaso. En el caso de Twitter, nunca ha habido intención de adaptar los mensajes a las características de esta red social, con lo que resulta lógico que el interés despertado sea menor. La mejoría vendría también con un seguimiento mucho más pormenorizado de los periodos de expediciones, que aumente la viralidad de este perfil.

En el caso de YouTube, los suscriptores son escasos pero, sin embargo, las reproducciones de los vídeos han sido todo un éxito. Esto indica que la labor de apoyo de YouTube a los perfiles de Facebook y Twitter, sobre todo el primero, sí que ha sido fructífera. Sólo podemos apuntar una posible mejoría en esta red social a través de la mayor calidad del formato de los vídeos, que podría suponer un incremento no sólo de suscriptores, sino también de seguidores en otras redes sociales.

\section{REFERENCIAS}

http://www.youtube.com/yosuconcarlossoria

http://www.facebook.com/yosuboconcarlossoria

http://www.twitter.com/retocarlossoria

http://prensa.bbva.com/informacion-corporativa/patrocinios/\%2825-9905\%29.html https://ngo.bbvasuma.com/user/bbva

\section{Juan Enrique Gonzálvez Vallés}

Profesor del centro de estudios Formatik, asociado a la Universidad Camilo José Cela. Miembro del Grupo de Investigación Concilium de la Universidad Complutense de Madrid. Licenciado en Periodismo con Diploma de Estudios Avanzados. Profesionalmente ha ejercido como periodista en diversos medios de comunicaciones y entidades. 